\title{
El screening auditivo neonatal en tres etapas con otoemisiones acústicas sería la estrategia más costo-efectiva
}

\section{Objetivo}

Comparar la costo-efectividad de diversas estrategias para el screening auditivo neonatal.

\section{Diseño}

Estudio de costo-efectividad con un modelo de análisis de decisiones, ${ }^{*}$ incluyendo un análisis de sensibilidad. ${ }^{*}$

\section{Lugar \\ Holanda.}

\section{Población objetivo}

Todos los infantes recién nacidos no admitidos en la Unidad de cuidados intensivos neonatal.

\section{Estrategias de rastreo a comparar}

Las estrategias evaluadas fueron definidas en base a método de screening (PEATC: Potenciales Evocados Auditivos del Tronco Cerebral u OEA: Otoemisiones Acústicas); número de etapas (visitas) en el proceso de screening (2 o 3); desorden blanco (pérdida de la audición bilateral o pérdida tanto uni como bilateral); lugar de la realización (hogar u hospital).

\section{Medición de resultados principales}

Costo por niño detectado con una pérdida de la audición $=40 \mathrm{db}$ en el mejor oído.

\section{Resultados principales}

El costo del proceso de screening en tres etapas en niños en el hospital fue de $£ 39$ (IC95\% 20-57) por niño detectado con PEATC vs. £25 (IC95\% 14,4-35,6) con OEA. El proceso de screening en 3 etapas redujo no sólo la tasa de derivación a estudios posteriores (debido a la menor tasa de falsos positivos) sino también los costos, en comparación con el screening en 2 etapas. El costo extra adicional de pasar de un programa de screening de enfermedad bilateral a uno unilateral osciló entre 1500-4000. Con la información disponible para la realización del estudio, no podría ser expresada la preferencia para una localización del screening particular (hogar u hospital).

\section{Conclusiones}

El estudio sugiere que el screening en tres etapas con OEA es la estrategia más costo-efectiva. Aunque el screening en el hogar parecería más costo-efectivo que el screening en el hospital se necesitan estudios posteriores.

\section{Comentario}

La prevalencia de hipoacusia en el recién nacido y el lactante se estima entre 1,5 y 6 casos por 1.000 nacidos vivos (según el grado). La detección temprana de sordera o pérdida auditiva severa, seguida de una intervención temprana, es importante para optimizar el desarrollo, especialmente en el lenguaje, en el habla y en las habilidades cognitivas.

La valoración subjetiva de la audición es difícil y poco sensible como método de cribado durante los primeros meses de vida.

De las pruebas objetivas de audición (electrofisiológicas), dos son las consideradas más útiles: Ios PEATC y OEA. Los potenciales auditivos tienen una sensibilidad del $97-100 \%$ y una especificidad del $86-96 \%$, y evalúan la actividad de la vía auditiva desde el nervio auditivo en su extremo distal hasta el mesencéfalo. Las OEA tiene una tasa de concordancia global con los PEATC de un $91 \%$, con una sensibilidad del $95 \%$ y una especificidad del $85 \%$ frente a los PEATC.

Hay un consenso generalizado en la recomendación del cribado auditivo neonatal en grupos de riesgo..$^{1-4}$ En cuanto al rastreo universal, las principales asociaciones y fuerzas preventivas lo aconsejan, con la notable excepción de la fuerza de tareas preventiva de EE.UU (U.S. Preventive Service Task Force), ${ }^{5}$ que no se pronuncia ni a favor ni en contra del rastreo universal por falta de evidencias suficientes (recomendación tipo I).

En ausencia de un programa de cribado universal neonatal, deben identificarse los niños con indicadores asociados a sordera (tanto congénita como adquirida) y asegurarse de que se realice en ellos una prueba objetiva cuanto antes. En el resto de los niños debe preguntarse a la familia sobre su audición, explorar la reacción al sonido y seguir de cerca la adquisición del lenguaje verbal. Una nota de cautela se refiere a la adaptación de los resultados de estudios de costo-efectividad a otros contextos en los que fueron realizados. El presente estudio fue realizado para Holanda, y la aplicabilidad de sus resultados a nivel de nuestros países no es directamente extrapolable.

\section{Conclusiones del comentador:}

Algunas cosas a tener en cuenta del presente estudio son: 1) el screening fue realizado con niños que no requirieron cuidado intensivo neonatal, 2) el costo del screening fue mayor con PEATC que con OEA debido principalmente al equipamiento requerido, 3) el screening en 3 etapas fue menos costoso. Aunque en una primera etapa aumenta ligeramente los costos, disminuye con futuras pruebas diagnósticas, 4) el screening para evaluar pérdida auditiva bilateral reduce tanto los costos como el número de niños referidos a estudios posteriores en el futuro $\left.{ }^{6}, 4\right)$ el screening con OEA en tres etapas (y posiblemente en el hogar) sería el más recomendable, aunque debería evaluarse la aplicabilidad de los resultados a nivel local.

\section{Dr. Sergio M. Polla [ Unidad de Medicina Familiar y Salud Comunitaria.Hospital Menor de Ing. White. Bahía Blanca. ]}

Polla S. El screening auditivo neonatal en tres etapas con Otoemisiones Acústicas sería la estrategia más costo-efectiva. Evid. actual. pract. ambul. 2004:7:172. Comentado de: Costs of different strategies for neonatal hearing screening: a modelling approach. Boshuizen, HC; van der Lem, GJ; Kauffman-de Boer, MA. Arch. Child Fet and Neonatal Edition. 2001;85(3):F177-181.

\section{Referencias}

1. Recomendations for Preventive Pediatric Health Care. American Academy Pediatrics. 2000

2. Canadian Task Force on the Periodic Health Examination. Canadian guide to clinical preventive health care. Ottawa: Canada Communication Group, 1994:258-266, 298-304, 954-963. 3. Joint Committee on Infant Hearing. Joint Committee on Infant Hearing 1994 position statement. Pediatrics 1995;95: 152-156.

4. Lutman ME, Grandori F. Screening for neonatal hearing defects European consensus statement. Eur J Pediatr 1999;158:95-96.

5. Di Giuseppi C, ed, Atkins D, ed, Woolf SH, ed. US Preventive Services Task Force Guide to Clinical Preventives Services. 2nd ed. Alexandria, Va: International Medical Publishing Inc; 1996

6. Norton SJ, Vohr B, Gorga MP, et al. A comparison of the effectiveness of three neonatal hearing screening procedures: auditory abrainstem responses (ABR), transient evoked otoacoustic emissions (TEOAE) and distortion product otoacoustic emissions (DPOAE). Pediatrics 1998;102:766. 